\title{
Model-based Active Damping Control for Three-Phase Voltage Source Inverters with LCL Filter
}

\author{
Ramon Guzman, Luis Garcia de Vicuña, Javier Morales, Miguel Castilla and Jaume Miret, Member, IEEE
}

\begin{abstract}
This paper presents a robust model-based active damping control in natural frame for a three-phase voltage source inverter with LCL filter. The presence of the LCL filter complicates the design of the control scheme, particularly when system parameters deviations are considered. The proposed control method is addressed to overcome such difficulties and uses a modified converter model in an state observer. In this proposal, the converter model is modified by introducing a virtual damping resistor. Then, a Kalman filter makes use of this model to estimate the system state-space variables. Although the state estimates do not obviously match the real world system variables, they permit designing three current sliding-mode controllers that provide the following features to the closed loop system: a) robust ande active damping capability like in the case of using a physical damping resistor, b) robustness because the control specifications are met independently of variation in the system parameters, c) noise immunity due to the application of the Kalman filter, and d) power loss minimization because the system losses caused by the physical damping resistor are avoided. An interesting side effect of the proposed control scheme is that the sliding surfaces for each controller are independent. This decoupling property for the three controllers allows using a fixed switching frequency algorithm that ensures perfect current control. To complete the control scheme, a theoretical stability analysis is developed. Finally, selected experimental results validate the proposed control strategy and permit illustrating all its appealing features.
\end{abstract}

Index Terms-Current control, LCL filter, Sliding Mode Control, Extended Kalman filter, Voltage sensorless.

\section{INTRODUCTION}

$\mathbf{V}$ OLTAGE source inverters (VSI's) are commonly used as interfaces between renewable energy sources and the utility grid. The main objective is to improve the overall grid stability, reliability and efficiency. The main function of the VSI is to extract the maximum power from the energy source and inject it to the grid as active power. Some amount of reactive power can also be injected according to specific requirements.

In VSIs with LCL filter, the controller design is a difficult task due to the inherent resonance problem which degrades the quality of the injected active power. Different solutions have been carried out in order to mitigate these oscillations using active or passive damping methods [1]-[4]. Passive damping methods are usually based on introducing a physical damping resistor in series with the capacitor filter. This option is simple but the overall system efficiency is reduced due to the losses [5]. These losses may be unacceptable in some applications as for instance wind turbines which operates typically at $30 \%$ of the converter nominal power [6]. As an alternative active damping methods can mitigate the oscillations without the use of physical damping resistors, thus reducing the system losses and improving the overall system efficiency. Several methods have been presented in the literature which can be classified in two main groups. The first method is based on the virtual resistor concept [7]-[11]. This algorithm uses a feedback current control in order to emulate a virtual resistor in the LCL filter. The main problem of this method is that extra sensors are needed for the controller design,in order to measure the filter capacitor current or voltage. The second method is the so-called filter-based active damping. Here, the control algorithm is modified by introduction of a digital filter in the closed-loop system in order to damp the highfrequencies [12]-[15]. This filter is correctly tuned ensuring the poles of the closed-loop system are inside of the unity circle in the z-plane. Usually low pass filters and notch filters are used but also lead-lag networks can be employed [16]. The effectiveness of this method relies on the accuracy in the filter tuning parameters in order to eliminate the resonance frequency.

However, variations in the system parameters or changes in the grid impedance become in variations of the the resonance frequency nominal value, and as a consequence a detuning problem in the filter may appear. Under these conditions, the uncertainty of the grid impedance at the point of common coupling (PCC), and also deviations in the system parameters are important concerns to be addressed. The aforementioned problems directly affect the resonance frequency value of the $L C L$ filter and might lead the current controller to an unstable dynamics due to a detuning of the digital filter.

In order to avoid self-tuning procedures, a new methodology based on sliding-mode control (SMC) using a state observer is presented in this paper. The main advantage of using the SMC technique, compared for instance with other proportionalresonant (P-RES) techniques, is that the controller bandwidth is increased. Here, it is important to remark that an oversample of the inverter-side current is necessary in order to operate at fixed switching frequency. This fact may limit the maximum switching frequency if a fast digital signal processor or Field Programmable Gate Array (FPGA) is not used. Besides, the current controller operates by comparing the current samples with a determined thresholds. Therefore, enough signal to noise ratio (SNR) is mandatory for the correct operation of the current controller [17].

A Kalman filter (KF) is then selected, instead of a standard 
state-observer, to estimate the system state variables in order to increase the system SNR and consequently the controller bandwidth. The computational time of the KF algorithm has been reduced in order to achieve a $4 \mathrm{kHz}$ switching frequency. Unlike the typical observer-based applications where an accurate converter model is considered [18],this proposal is based on modifying the converter model in order to obtain active damping capabilities. It will show that the use of modified models in observer-based techniques may become a benefit in the control performances. This is an interesting feature of the proposed control system that provides an inherent active damping to the conventional SMC. Another interesting feature of this proposal is that the proposed model does not rely on the grid inductance value, which improves the robustness against this parameter. Besides, the proposed control algorithm does not use any self-tuning procedure or adaptive techniques to provide robustness against parameters deviations, even in the case of severe changes in the grid impedance.

In order to analyze and design the proposed robust controller the following steps will be taken:

1) A modified model of the converter model is derived. This model has two important features: first, the model contains a damping term and second, the model does not rely on the grid inductance value.

2) A conventional switching surface is proposed using estimated variables obtained from the observer.

3) A complete stability analysis is performed taking into account the effect of the KF, the system discretization and the deviation in the system parameters. This analysis is addressed to prove the system robustness.

4) Finally, selected experimental results are reported to prove the aforementioned properties, including the controller response against grid voltage unbalance and distortion disturbances.

Using the proposed method, the main advantages of the control algorithm are: 1) the active damping is achieved without using digital filters in the closed loop system, thus avoiding detuning problems, for instance in the case of grid inductance variations. Note that the KF is used as a stateobserver and not as a filter to eliminate the resonance, 2) no extra sensors are needed,as in the case of virtual resistor methods, 3) the system is robust against system parameters deviation, and 4) the system exhibits a high immunity to noise due to the KF application.

The paper is organized as follows. In Section II the statespace equation for the converter model is presented. Section III describes the problem formulation. Section IV introduces the proposed control system. In Section V the stability analysis is presented. Section VI presents some simulation results of a virtual resistor method. In section VII the experimental results are reported. Finally, section VIII concludes the paper.

\section{DeRivation of THE STATE-SPACE EQUATIONS FOR THE VSI WITH LCL FILTER}

The three-phase system equations can be obtained from the circuit shown in Fig.1 as :

$$
\begin{aligned}
L_{1} \frac{d \mathbf{i}_{1}}{d t} & =\frac{V_{d c}}{2} \mathbf{u}-\mathbf{v}_{c}-v_{n} \mathbf{1} \\
C \frac{d \mathbf{v}_{c}}{d t} & =\mathbf{i}_{1}-\mathbf{i}_{2} \\
\left(L_{2}+L_{g}\right) \frac{d \mathbf{i}_{2}}{d t} & =\mathbf{v}_{c}+\left(v_{n}-v_{n}^{\prime}\right) \mathbf{1}-\mathbf{v}_{g}
\end{aligned}
$$

where $\mathbf{i}_{1}=\left[\begin{array}{lll}i_{1 a} & i_{1 b} & i_{1 c}\end{array}\right]^{T}$ is the inverter-side current vector, $\mathbf{i}_{2}=\left[\begin{array}{lll}i_{2 a} & i_{2 b} & i_{2 c}\end{array}\right]^{T}$ is the grid-side current vector, $\mathbf{v}_{c}=\left[\begin{array}{lll}v_{c a} & v_{c b} & v_{c c}\end{array}\right]^{T}$ is the capacitor voltage vector, $\mathbf{v}_{g}=$ $\left[\begin{array}{lll}v_{g a} & v_{g b} & v_{g c}\end{array}\right]^{T}$ is the grid voltage vector, $\mathbf{u}=\left[\begin{array}{lll}u_{a} & u_{b} & u_{c}\end{array}\right]^{T}$ is the control signal vector, $v_{n}$ and $v_{n}^{\prime}$ are the voltages at the neutral points and $\mathbf{1}$ is a column vector defined as $\left[\begin{array}{lll}1 & 1 & 1\end{array}\right]^{T}$. Using (1) and (3) the equations for the neutral point voltages can be found as

$$
\begin{gathered}
v_{n}=\frac{V_{d c}}{6}\left(u_{a}+u_{b}+u_{c}\right)-\frac{1}{3}\left(v_{c a}+v_{c b}+v_{c c}\right) \\
v_{n}^{\prime}=\frac{V_{d c}}{6}\left(u_{a}+u_{b}+u_{c}\right)-\frac{1}{3}\left(v_{g a}+v_{g b}+v_{g c}\right) .
\end{gathered}
$$

Assuming that the grid voltages are balanced (i.e., $v_{g a}+v_{g b}+$ $v_{g c}=0$ and $v_{c a}+v_{c b}+v_{c c}=0$ ), the expressions for the neutral point voltages are reduced to

$$
v_{n}=v_{n}^{\prime}=\frac{V_{d c}}{6}\left(u_{a}+u_{b}+u_{c}\right)
$$

and taking into account (6) the new expressions for the threephase system can be written as

$$
\begin{aligned}
L_{1} \frac{d \mathbf{i}_{1}}{d t} & =\frac{V_{d c}}{2} \mathbf{u}-\mathbf{v}_{c}-v_{n} \mathbf{1} \\
C \frac{d \mathbf{v}_{c}}{d t} & =\mathbf{i}_{1}-\mathbf{i}_{2} \\
\left(L_{2}+L_{g}\right) \frac{d \mathbf{i}_{2}}{d t} & =\mathbf{v}_{c}-\mathbf{v}_{g} .
\end{aligned}
$$

The above differential equations can be rewritten as a discrete state-space model. Then, the process and measurement discrete equations for each phase-leg $i$ are expressed as follows:

$$
\begin{gathered}
\mathbf{x}_{i}(k+1)=\mathbf{A} \mathbf{x}_{i}(k)+\mathbf{B} u_{i}(k)+\mathbf{D} v_{i}(k)+\mathbf{E} v_{n}(k) \\
y_{i}(k)=\mathbf{H} x_{i}(k)
\end{gathered}
$$

where $\mathbf{x}_{i}(k)=\left(\begin{array}{lll}i_{1 i} & v_{c i} & i_{2 i}\end{array}\right)$ is the state-space vector, $\mathbf{H}$ is the output matrix defined as

$$
\mathbf{H}=\left(\begin{array}{lll}
1 & 0 & 0
\end{array}\right)
$$

and the discrete matrices $\mathbf{A}, \mathbf{B}, \mathbf{D}$ and $\mathbf{E}$ are computed using the first order approximation as follows

$$
\begin{gathered}
\mathbf{A} \cong \mathbf{I}+\mathbf{A}_{c} T_{s}=\left(\begin{array}{ccc}
1 & -T_{s} / L_{1} & 0 \\
T_{s} / C & 1 & -T_{s} / C \\
0 & T_{s} /\left(L_{2}+L_{g}\right) & 1
\end{array}\right) \\
\mathbf{B} \cong \mathbf{B}_{c} T_{s}=\left(\begin{array}{lll}
V_{d c} T_{s} / 2 L_{1} & 0 & 0
\end{array}\right)^{T} \\
\mathbf{D} \cong \mathbf{D}_{c} T_{s}=\left(\begin{array}{ccc}
0 & 0 & -T_{s} /\left(L_{2}+L_{g}\right)
\end{array}\right)^{T}
\end{gathered}
$$




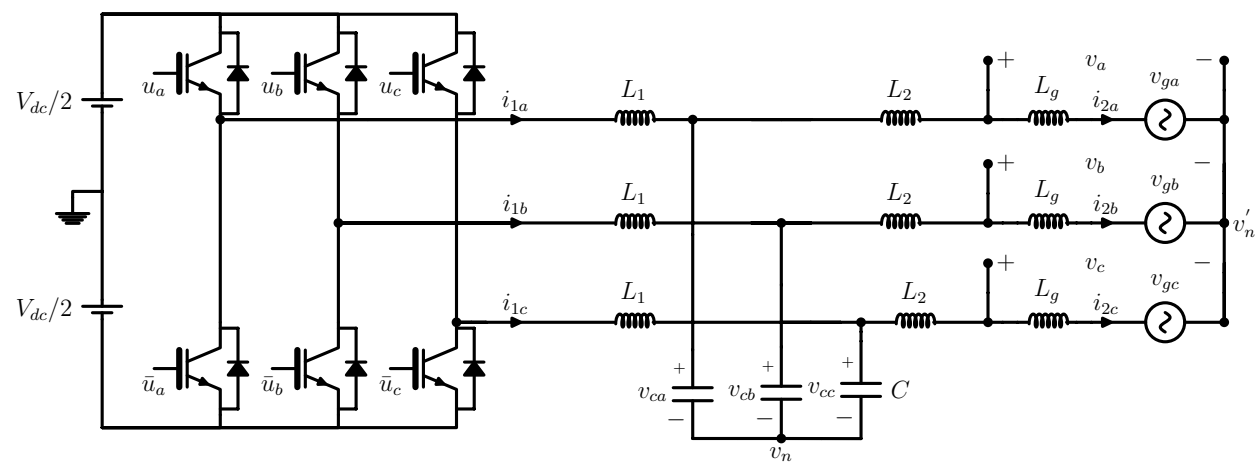

Fig. 1. Circuit diagram of three-phase grid-connedcted inverter with LCL-filter

$$
\mathbf{E} \cong \mathbf{E}_{c} T_{s}=\left(\begin{array}{lll}
-T_{s} / L_{1} & 0 & 0
\end{array}\right)^{T}
$$

being $T_{s}$ the sampling time and $\mathbf{A}_{c}, \mathbf{B}_{c}, \mathbf{D}_{c}$ and $\mathbf{E}_{c}$ the continuous system matrices. These discrete equations will be used in the stability analysis section in order to validate our control proposal.

\section{PROBLEM FORMULATION}

In this section, the $L C L$ resonance problem when the conventional SMC is used to control the VSI is presented. Fig. 1 shows the circuit scheme of the VSI which uses an LCL filter without physical damping resistor.

Typically, the sliding surfaces are generated using the inverter side currents as follows:

$$
\mathbf{S}=\mathbf{i}_{1}-\mathbf{i}^{*}
$$

where $\mathbf{i}^{*}=\left[\begin{array}{lll}i_{a}^{*} & i_{b}^{*} & i_{c}^{*}\end{array}\right]$ is the reference current vector which can be obtained from the reference active and reactive powers as in [19]:

$$
\begin{aligned}
i_{a}^{*} & =\frac{P^{*}}{|\mathbf{v}|^{2}} v_{a}+\frac{Q^{*}}{\sqrt{3}|\mathbf{v}|^{2}}\left(v_{b}-v_{c}\right) \\
i_{b}^{*} & =\frac{P^{*}}{|\mathbf{v}|^{2}} v_{b}+\frac{Q^{*}}{\sqrt{3}|\mathbf{v}|^{2}}\left(v_{c}-v_{a}\right) \\
i_{c}^{*} & =-\left(i_{a}^{*}+i_{b}^{*}\right)
\end{aligned}
$$

with $|\mathbf{v}|^{2}=v_{a}^{2}+v_{b}^{2}+v_{c}^{2}$ and $P^{*}$ and $Q^{*}$ are the active and reactive power, respectively.

Assuming that the system is in sliding regime (i.e. $\mathbf{S}=\dot{\mathbf{S}}=$ $0)$, the inverter-side current is forced to track its reference. Then, the differential equations (7)-(9) can be rewritten as follows:

$$
\begin{aligned}
\mathbf{i}_{1} & =\mathbf{i}^{*} \\
C \frac{d \mathbf{v}_{c}}{d t} & =\mathbf{i}^{*}-\mathbf{i}_{2} \\
\left(L_{2}+L_{g}\right) \frac{d \mathbf{i}_{2}}{d t} & =\mathbf{v}_{c}-\mathbf{v}_{g}
\end{aligned}
$$

Now from (21)-(23) the differential equation for the gridside current dynamics can be found

$$
\left(L_{2}+L_{g}\right) C \frac{d^{2} \mathbf{i}_{2}}{d t^{2}}+\mathbf{i}_{2}=\mathbf{i}^{*}-C \frac{d \mathbf{v}_{g}}{d t} .
$$

As it can be deduced from (24), the grid-side current dynamic is oscillatory (due to the absence of the first-time derivative of $i_{2}$ in this equation), and the resonance frequency is given by:

$$
f_{\text {res }}=1 / 2 \pi \sqrt{\left(L_{2}+L_{g}\right) C}
$$

From the last expression, the resonance frequency is only given by the filter parameters $L_{2}$ and $C$ and also by the grid inductance $L_{g}$. This fact is due to that the SMC forces the inverter-side current $\mathbf{i}_{1}$ to track its reference and the system order is reduced in one. Equation (25) shows that variations in the grid inductance leads to variations in the resonance frequency value, which complicates the design of the controller.

\section{Proposed Control System}

The proposed control scheme for phase-leg $a$ is depicted in Fig.2. The controller for remaining phases has the same block diagram. The controller scheme is addressed to stabilize the system and also to improve the robustness against system parameters uncertainties, specially variations in the grid inductance. To deal with this specifications, the following control algorithm is presented.

An modified model of the VSI is used in an state observer, in our case a KF. According with the control specifications, a virtual damping resistor has been added in the model as shown in matrix (35). Besides, the converter state-space model is augmented in two additional states, the voltage at the PCC and its quadrature [20]. This new state variables allows to obtain a sinusoidal reference current even in the case of a highly distorted grid. Then, according to Fig.2, the reference current can be obtained from the estimated PCC voltages and the desired reference active power.

Once the inverter-side current is estimated and the reference current is computed both signals are used in a sliding surface which computes the error between them. Then, a switching algorithm computes the control signal according to this error. This algorithm is presented in [21] and it is used to improve the switching spectrum of the SMC. Finally, to validate this control proposal a complete stability analysis including the system discretization and the observer effect is performed.

\section{A. Proposed state-space model of the converter}

This section deals with the converter model deduction in order to be used in the KF algorithm. The main objective 


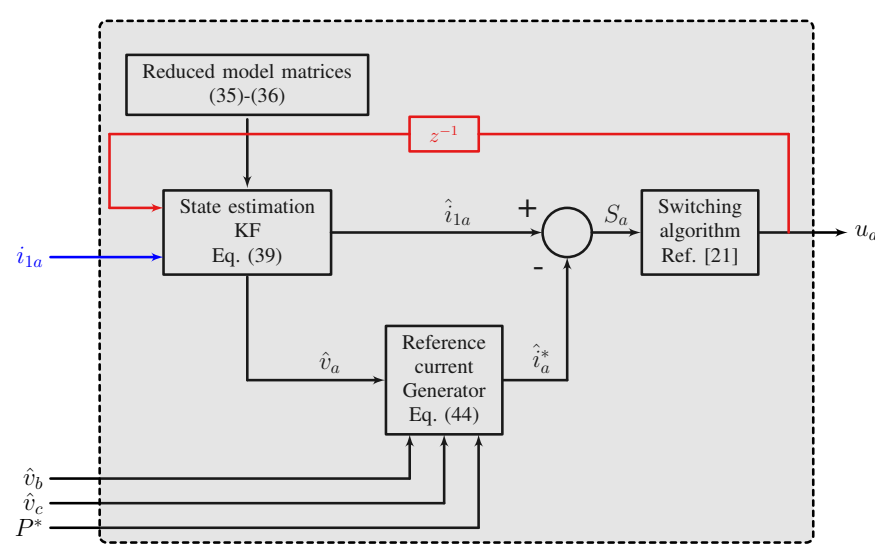

Fig. 2. Proposed control system for phase-leg $a$

is to obtain three decoupled controllers with active damping capabilities. It must be noticed in (10) the influence of the neutral point voltage. This term can provoke severe interferences between controllers, thus causing severe irregularities in the ordinary hysteresis operation. In order to eliminate these interferences, a decoupled model is proposed by removing the neutral point voltage from the converter model in (7). This fact has a negligible effect in the low-frequency dynamics of the closed-loop system. The reason is that $v_{n}$ is a high-frequency signal with nearly zero average value; see (6). Besides the VSI model has been modified with two objectives in mind: 1) to achieve active damping capabilities by adding a damping resistor $R_{d}$ in series with the filter capacitor, and 2) to estimate the fundamental component and its quadrature of the PCC voltage by adding two additional states. According to [20], this fact will allow to generate sinusoidal reference currents even in case of a distorted grid. With these assumptions the proposed model can be expressed as:

$$
\begin{aligned}
L_{1 o} \frac{d \mathbf{i}_{1}}{d t} & =\frac{V_{d c}}{2} \mathbf{u}-R_{d}\left(\mathbf{i}_{1}-\mathbf{i}_{2}\right)-\mathbf{v}_{c} \\
C_{o} \frac{d \mathbf{v}_{c}}{d t} & =\mathbf{i}_{1}-\mathbf{i}_{2} \\
L_{2 o} \frac{d \mathbf{i}_{2}}{d t} & =R_{d}\left(\mathbf{i}_{1}-\mathbf{i}_{2}\right)+\mathbf{v}_{c}-\mathbf{v} \\
\frac{d \mathbf{v}}{d t} & =\omega_{o} \mathbf{v}_{q} \\
\frac{d \mathbf{v}_{q}}{d t} & =-\omega_{o} \mathbf{v}
\end{aligned}
$$

being $L_{1 o}, L_{2 o}, C_{o}$ the nominal values of the VSI parameters, $\omega_{o}$ the grid angular frequency, and $\mathbf{v}$ and $\mathbf{v}_{q}$ the voltage at the PCC and its quadrature respectively [21].

This model is discretized in order to be used in the KF algorithm. The augmented discrete state -space model which includes the PCC voltages is written as follows:

$$
\begin{aligned}
\mathbf{x}_{i, a u g}(k+1) & =\hat{\mathbf{A}}_{\text {aug }} \mathbf{x}_{i, a u g}(k)+\mathbf{B}_{\text {aug }} u_{i}(k)+\boldsymbol{\eta}_{i}(k) \\
y_{i}(k) & =\mathbf{H}_{\text {aug }} x_{i}(k)+\mathbf{w}_{i}(k)
\end{aligned}
$$

where $\boldsymbol{\eta}_{i}(k)$ and $\mathbf{w}_{i}(k)$ are the process and the measurement noise vectors respectively, which defines the following noise and process covariance matrices:

$$
\begin{aligned}
\mathbf{R}_{i}(k) & =E\left\{\mathbf{w}_{i}(k) \mathbf{w}_{i}^{T}(k)\right\} \\
\mathbf{Q}_{i}(k) & =E\left\{\boldsymbol{\eta}_{i}(k) \boldsymbol{\eta}_{i}^{T}(k)\right\}
\end{aligned}
$$

and $\hat{\mathbf{A}}_{\text {aug }}$ is the augmented the transition matrix of the proposed converter model, in which, a damping resistor has been introduced, and given by:

$$
\begin{aligned}
& \hat{\mathbf{A}}_{a u g} \cong\left(\begin{array}{ccc}
1-T_{s} R_{d} / L_{1} & -T_{s} / L_{1 o} & T_{s} R_{d} / L_{1 o} \\
T_{s} / C_{o} & 1 & -T_{s} / C_{o} \\
T_{s} R_{d} / L_{2 o} & T_{s} / L_{2 o} & 1-T_{s} R_{d} / L_{2 o} \\
0 & 0 & 0 \\
0 & 0 & 0
\end{array}\right. \\
& \left.\begin{array}{cc}
0 & 0 \\
0 & 0 \\
-T_{s} / L_{2 o} & 0 \\
0 & T_{s} \omega_{o} \\
-T_{s} \omega_{o} & 0
\end{array}\right)
\end{aligned}
$$

The remaining matrices are

$$
\begin{aligned}
& \mathbf{B}_{a u g} \cong\left(\begin{array}{lllll}
V_{d c} T_{s} / 2 L_{1 o} & 0 & 0 & 0 & 0
\end{array}\right)^{T} \\
& \mathbf{H}_{\text {aug }}=\left(\begin{array}{lllll}
1 & 0 & 0 & 0 & 0
\end{array}\right)
\end{aligned}
$$

being the augmented state-space vector

$$
\mathbf{x}_{i}(k)=\left(\begin{array}{lllll}
i_{1 i} & v_{c i} & i_{2 i} & v_{i} & v_{i q}
\end{array}\right)
$$

\section{B. Kalman filter adaptive equation}

The proposed method is based on the state-space model defined by (31)-(32). If this model is used in a KF, the estimated variables will have similar dynamics as when a physical damping resistor is used. Then, these estimated variables can be used as inputs of the sliding surfaces providing the three independent controllers with active damping capabilities. The main properties and the implementation of the KF are well explained in [22] so only the adaptive equation used in the estimation will be given in this section.

Based on the KF algorithm implementation, the equation for the estate estimation can be expressed as follows:

$$
\begin{aligned}
\hat{\mathbf{x}}_{i, a u g}(k+1) & =\hat{\mathbf{A}}_{\text {aug }} \hat{\mathbf{x}}_{i}(k)+\mathbf{B}_{\text {aug }} u_{i}(k) \\
& +\mathbf{L}_{i, a u g}(k)\left(\mathbf{x}_{i, a u g}(k)-\hat{\mathbf{x}}_{i, a u g}(k)\right)
\end{aligned}
$$

where the Kalman gain is computed as:

$$
\mathbf{L}_{i, a u g}(k)=\mathbf{P}_{i}(k) \mathbf{H}_{\text {aug }}^{T}\left(\mathbf{H}_{\text {aug }} \mathbf{P}_{i}(k) \mathbf{H}_{\text {aug }}^{T}+\mathbf{R}_{i}(k)\right)^{-1} .
$$

1) Consideration in the Kalman gain computation: A high computational load is usually the main drawback in a $\mathrm{KF}$ algorithm implementation. Specially, (40) contains a matrix inversion which involves a long computational time. Using a KF for each phase-leg instead a single KF for the whole three-phase system, the matrix inversion of (40) is reduced to a scalar inversion. Actually, according to (37) and taking into account that $\mathbf{P}_{i}(k)$ is a square matrix, the computation of $\mathbf{H}_{\text {aug }} \mathbf{P}_{i}(k) \mathbf{H}_{\text {aug }}^{T}$ in the Kalman gain expression results 
in a scalar. For this reason and to be consistent with the matrix addition in (40), the noise covariance matrix $\mathbf{R}_{i}(k)$ is considered as the single-phase mean noise power, $R_{i}(k)$. Besides, this noise is so similar in each phase leg and the Kalman gain can be assumed identical for all the phases (i.e. $\left.\mathbf{L}_{a, a u g}(k) \cong \mathbf{L}_{b, a u g}(k) \cong \mathbf{L}_{c, a u g}(k)\right)$. Thanks to this approximation, (40) is computed only for one phase and used in all the phases. Following this approach a notably reduction in the KF implementation is practically obtained.

2) Mean noise power estimation: The value of $R_{i}(k)$ can be estimated using the unbiased mean power estimator

$$
R_{i}(k)=\frac{1}{N T_{s}} \sum_{k=0}^{N-1}\left|w_{i}(k)\right|^{2}
$$

where $w_{i}(k)$ is an additive white gaussian noise sample at time $k$, in phase-leg $i$, and $N$ is the number of samples.

To obtain $w_{i}(k)$, the simplest way is to use a dc-voltage contaminated with gaussian white noise. In this case a reference dc-voltage used in the sensing system $V_{r e f}$ is employed. This voltage is connected at an analog input of the DSP and is contaminated with the system noise when the inverter is switching. The noise samples can be obtained by subtracting from the $V_{\text {ref }}$ measures, the exact value, $V_{\text {ref }}^{*}$ (i.e. the measured value when the inverter is not switching), yielding

$$
R_{i}(k)=\frac{1}{N T_{s}} \sum_{k=0}^{N-1}\left|V_{r e f}(k)-V_{r e f}^{*}(k)\right|^{2}
$$

The last expression allows to estimate the mean noise power in any of the three phases and can be used to compute the Kalman gain. Assuming the noise power level has no important variations, $R_{i}(k)$ is computed at the beginning and remains unchanged during all the time.

3) Process covariance matrix: Matrix $\mathbf{Q}_{i}(k)$ is not so straightforward to be found. It is generally derived intuitively, but there are some points that need to be regarded in its selection [22]. Unmodeled dynamics and parameter uncertainties are generally modeled as process noise. Usually $\mathbf{Q}_{i}(k)$ is taken as a diagonal matrix which in this application is of dimension 5. Its value has been obtained by means of simulations. An approximate value of $\mathbf{Q}_{i}(k)=0.005 \mathbf{I}_{5}$, where $\mathbf{I}_{5}$ is a 5dimensional unity matrix, is obtained which provides a good behaviour.

\section{Sliding surfaces with active damping capability}

The model-based control solution proposed in this paper is designed to provide active damping capabilities to the controllers. In order to achieve this damping performance, the model defined by $(26)-(30)$ is used in a KF algorithm providing an estimated state of the inverter-side current, with similar dynamics performance as when a physical damping resistor is used. To achieve this objective, the controllers use the estimated inverter-side current as follows:

$$
S_{i}=\mathbf{C}^{T} \hat{\mathbf{x}}_{i, a u g}-\hat{i}_{i}^{*}=\hat{i}_{1 i}-\hat{i}_{i}^{*}
$$

where $\mathbf{C}^{T}=\left(\begin{array}{ccccc}1 & 0 & 0 & 0 & 0\end{array}\right)$, and $\hat{i}_{i}^{*}$ is the reference current obtained from the estimated PCC voltages, computed as:

$$
\begin{aligned}
& \hat{i}_{a}^{*}=\frac{P^{*}}{|\mathbf{v}|^{2}} \hat{v}_{a} \\
& \hat{i}_{b}^{*}=\frac{P^{*}}{|\mathbf{v}|^{2}} \hat{v}_{b} \\
& \hat{i}_{c}^{*}=-\left(\hat{i}_{a}^{*}+\hat{i}_{b}^{*}\right) .
\end{aligned}
$$

When the system is in ideal sliding regime (i.e. $\mathbf{S}_{i}=\dot{\mathbf{S}}_{\mathbf{i}}=0$ ), the estimated inverter-side current tracks its reference. Then, the differential equations for the estimated variables will accomplish:

$$
\begin{aligned}
\hat{\mathbf{i}}_{1} & =\hat{\mathbf{i}}^{*} \\
C_{o} \frac{d \hat{\mathbf{v}}_{c}}{d t} & =\hat{\mathbf{i}}_{1}-\hat{\mathbf{i}}_{2} \\
L_{2 o} \frac{d \hat{\mathbf{i}}_{2}}{d t} & =R_{d}\left(\hat{\mathbf{i}}_{1}-\hat{\mathbf{i}}_{2}\right)+\hat{\mathbf{v}}_{c}-\hat{\mathbf{v}} .
\end{aligned}
$$

Thus, from the last equations, the closed-loop estimated grid-side current dynamics can be derived in a similar way as in section III, yielding:

$$
L_{2 o} C_{o} \frac{d^{2} \hat{\mathbf{i}}_{2}}{d t^{2}}+R_{d} C \frac{d \hat{\mathbf{i}}_{2}}{d t}+\hat{\mathbf{i}}_{2}=\hat{\mathbf{i}}^{*}+R_{d} C \frac{d \hat{\mathbf{i}}^{*}}{d t}-C_{o} \frac{d \hat{\mathbf{v}}}{d t} .
$$

The last equation shows that the ideal sliding dynamics is stable. Note that the dynamics does not rely on the grid inductance value. This is an important difference with respect the dynamics expressed in (24). However, the previous analysis is not complete since the system discretization and the $\mathrm{KF}$ effect have not been taken into account. For this reason, a complete stability analysis including the KF should be performed in order to prove the overall system stability. This analysis will be introduced in section V.

\section{Stability ANALYSis}

The stability analysis is performed in this section to prove that the VSI using the proposed control method is stable. The validity is tested by ensuring the eigenvalues of the closed loop system are inside the unity circle in the z-plane. Besides a test of stability in the case of system parameters deviation will be provided in the last part of this section.

The three-phase system has nine poles, each one associated to one state variable. Thanks to the decoupling introduced by the KF with the proposed model, a decoupling between controllers is achieved and the three-phase system can be treated as three independent single-phase systems. This is the reason why only the stability of a single-phase system is analyzed in this section, and only three system poles are considered.

However, an state observer is used and the number of poles is duplicated. The system together with the observer have a total of six poles. While the system is in sliding regime, it is found that there is one pole fixed at the origin $(z=0)$ [23]. The position of the remaining poles can be adjusted by the parameter $R_{d}$. The next subsections are dedicated to find the stability equations. 


\section{A. Discrete equivalent control deduction}

This subsection focuses on finding the equivalent control expression. This expression will be used in the next subsection in order to obtain the closed-loop system equations for the analysis of the system stability.

Assuming the system is in sliding regime the control signal $u_{i}$ in (39) can be substituted by the so-called "equivalent control" $\hat{u}_{i e q}$ [24]. In addition the estimated voltages at the PCC can be considered as disturbances for the complete system. Thus, they can be removed from the system equations since they have no effect in the stability analysis. With these assumptions the reduced state-space model is as follows:

$$
\hat{\mathbf{x}}_{i}(k+1)=\hat{\mathbf{A}} \hat{\mathbf{x}}_{i}(k)+\mathbf{B} \hat{u}_{i e q}(k)+\mathbf{L}_{i}(k) \mathbf{H}\left(\mathbf{x}_{i}(k)-\hat{\mathbf{x}}_{i}(k)\right) .
$$

where $L_{i}(k)$ is the reduced Kalman gain containing only the three first values of $L_{i, a u g}(k)$, and

$$
\hat{\mathbf{A}} \cong\left(\begin{array}{ccc}
1-T_{s} R_{d} / L_{1 o} & -T_{s} / L_{1 o} & T_{s} R_{d} / L_{1 o} \\
T_{s} / C_{o} & 1 & -T_{s} / C_{o} \\
T_{s} R_{d} / L_{2 o} & T_{s} / L_{2 o} & 1-T_{s} R_{d} / L_{2 o}
\end{array}\right)
$$

Note that the aforementioned matrix is obtained from (35).

In order to obtain the equivalent control expression, the previous equation can be used in the sliding surface equation (43) at time $k+1$ which leads to

$$
\begin{aligned}
S_{i}(k+1) & =\mathbf{C}^{T} \hat{\mathbf{x}}_{i}(k+1)-\hat{i}_{i}^{*}(k+1) \\
& =\mathbf{C}^{T}\left(\hat{\mathbf{A}} \hat{\mathbf{x}}_{i}(k)+\mathbf{B} \hat{u}_{i e q}(k)\right. \\
& +\mathbf{L}_{i}(k) \mathbf{H}\left(\mathbf{x}_{i}(k)-\hat{\mathbf{x}}_{i}(k)\right)-\hat{i}_{i}^{*}(k+1)
\end{aligned}
$$

which leads to:

$$
\begin{aligned}
\hat{u}_{i e q}(k) & =\left(\mathbf{C}^{T} \mathbf{B}\right)^{-1}\left(\hat{i}_{i}^{*}(k+1)+S_{i}(k+1)\right. \\
& \left.-\mathbf{C}^{T} \hat{\mathbf{A}} \hat{\mathbf{x}}_{i}(k)-\mathbf{C}^{T} \mathbf{L}_{i}(k) \mathbf{H} \mathbf{e}_{i}(k)\right)
\end{aligned}
$$

being the error defined as

$$
\mathbf{e}_{i}(k)=\mathbf{x}_{i}(k)-\hat{\mathbf{x}}_{i}(k) .
$$

Assuming that the system is in sliding regime (i.e. $S_{i}(k+$ 1) $=S_{i}(k)=0$ ), the aforementioned equation can be rewritten as follows

$$
\hat{u}_{i e q}(k)=\left(\mathbf{C}^{T} \mathbf{B}\right)^{-1} \Delta \hat{i}_{i}^{*}+\mathbf{K}_{1} \hat{\mathbf{x}}_{i}(k)+\mathbf{K}_{2} \mathbf{e}_{i}(k)
$$

where

$$
\begin{aligned}
\Delta \hat{i}_{i}^{*} & =\hat{i}_{i}^{*}(k+1)-\hat{i}_{i}^{*}(k) \\
\mathbf{K}_{1} & =-\left(\mathbf{C}^{T} \mathbf{B}\right)^{-1} \mathbf{C}^{T} \hat{\mathbf{A}} \\
\mathbf{K}_{2} & =-\left(\mathbf{C}^{T} \mathbf{B}\right)^{-1} \mathbf{C}^{T} \mathbf{L}_{i}(k) \mathbf{H} .
\end{aligned}
$$

By substituting (57)-(59) in (56), the equivalent control can be expressed as follows:

$$
\begin{gathered}
\hat{u}_{i e q}(k)=\frac{2}{V_{d c}}\left[L_{1 o} \frac{\Delta \hat{i}_{i}^{*}}{T_{s}}+R_{d}\right. \\
\left.\left(\hat{i}_{1 i}(k)-\hat{i}_{2 i}(k)\right)+\hat{v}_{c i}(k)-L_{1} L_{k 1 i}(k)\left(i_{1 i}(k)-\hat{i}_{1 i}(k)\right)\right]
\end{gathered}
$$

where $L_{k 1 i}(k)$ is the first component of the Kalman gain vector for phase-leg $i$. The aforementioned expression shows

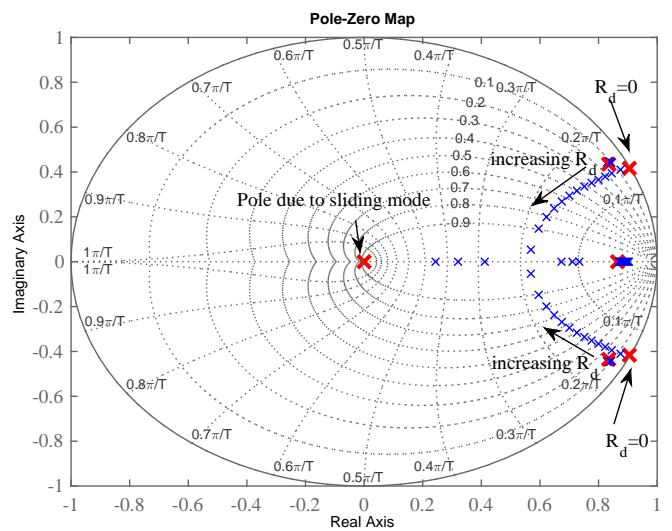

Fig. 3. Pole map for variations in $R_{d}$ from $0 \Omega$ up to $20 \Omega$.

clearly the influence of the virtual damping resistor and the gain observer in the equivalent control. Note that this expression not depends on the neutral point voltage (6) providing an effective decoupling between controllers.

\section{B. Closed-loop system equations}

The closed-loop system equations are derived in this section. These equations are defined by the vector $\left[\mathbf{x}_{i}(k) \mathbf{e}_{i}(k)\right]$, where $\mathbf{e}_{i}(k)$ is the error for each phase leg $i$ between the real statespace vector and the estimated one, as defined in (55).

The discrete state-space equations for the VSI, when estimated variables are used in the controller, can be defined as follows

$$
\mathbf{x}_{i}(k+1)=\mathbf{A} \mathbf{x}_{i}(k)+\mathbf{B} \hat{u}_{i e q}(k)
$$

Replacing (56) in (61) and (51) and taking into account (55), the following state-space equations for the real system variables and for the estimated ones can be obtained

$$
\begin{aligned}
\mathbf{x}_{i}(k+1) & =\left(\mathbf{A}+\mathbf{B} \mathbf{K}_{1}\right) \mathbf{x}_{i}(k)+\mathbf{B}\left(\mathbf{K}_{2}-\mathbf{K}_{1}\right) \mathbf{e}_{i}(k) \\
& +\mathbf{B}\left(\mathbf{C}^{T} \mathbf{B}\right)^{-1} \Delta \hat{i}_{i}^{*} \\
\hat{\mathbf{x}}_{i}(k+1) & =\left(\hat{\mathbf{A}}+\mathbf{B} \mathbf{K}_{1}\right) \mathbf{x}_{i}(k)+\left(\mathbf{L}_{i} \mathbf{H}+\mathbf{B}\left(\mathbf{K}_{2}-\mathbf{K}_{1}\right)\right. \\
& -\mathbf{A}) \mathbf{e}_{i}(k)+\mathbf{B}\left(\mathbf{C}^{T} \mathbf{B}\right)^{-1} \Delta \hat{i}_{i}^{*}
\end{aligned}
$$

By subtracting (63) from (62) the error in $k+1$ is obtained

$$
\mathbf{e}_{i}(k+1)=(\mathbf{A}-\hat{\mathbf{A}}) \mathbf{x}_{i}(k)+\left(\mathbf{A}-\mathbf{L}_{i} \mathbf{H}\right) \mathbf{e}_{i}(k) .
$$

Equations (62) and (64) define the equations for the closedloop system which can be written in matrix form

$$
\left[\begin{array}{l}
\mathbf{x}_{i}(k+1) \\
\mathbf{e}_{i}(k+1)
\end{array}\right]=\mathbf{G}\left[\begin{array}{l}
\mathbf{x}_{i}(k) \\
\mathbf{e}_{i}(k)
\end{array}\right]
$$

where

$$
\mathbf{G}=\left(\begin{array}{cc}
\mathbf{A}+\mathbf{B} \mathbf{K}_{1} & \mathbf{B}\left(\mathbf{K}_{2}-\mathbf{K}_{1}\right) \\
\mathbf{A}-\hat{\mathbf{A}} & \hat{\mathbf{A}}-\mathbf{L}_{i} \mathbf{H}
\end{array}\right)
$$




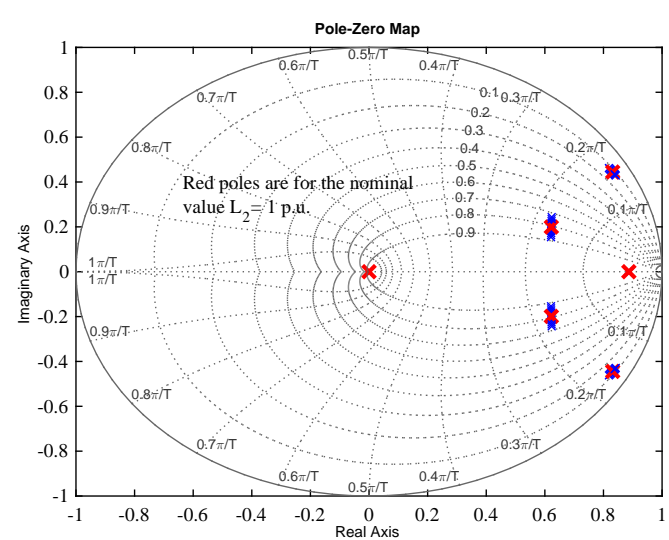

(a)

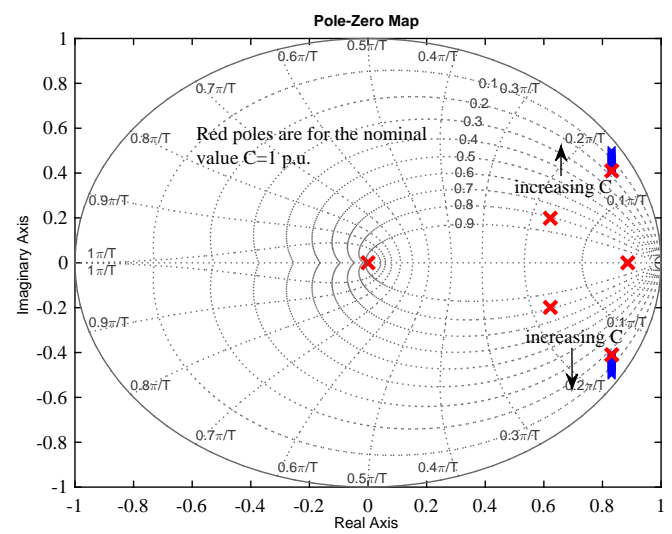

(b)

Fig. 4. Root locus diagrams when $R_{d}=10 \Omega$. a) $L_{2}$ vary $\pm 30 \%$. b) C vary $\pm 30 \%$.

The system stability will be given by the eigenvalues of the matrix in (66) which is the solution of the following equation with respect $\lambda$ :

$$
\begin{aligned}
& \left(\mathbf{A}+\mathbf{B K}_{1}-\lambda \mathbf{I}\right)\left(\hat{\mathbf{A}}-\mathbf{L}_{i} \mathbf{H}-\lambda \mathbf{I}\right) \\
& -\mathbf{B}\left(\mathbf{K}_{2}-\mathbf{K}_{1}\right)(\mathbf{A}-\hat{\mathbf{A}})=0 .
\end{aligned}
$$

By choosing the parameter $R_{d}=0 \Omega$ it is accomplished that $\hat{A}=\hat{\mathbf{A}}$ and the eigenvalues of (66) can be separated into poles due to the system and poles due to the observer. This affirmation can be clearly deduced if the relation $\mathbf{A}=\hat{\mathbf{A}}$ is used in (67). As evident some system poles are outside of the unity circle since no damping resistor, and as a result the active damping is not achieved.

Otherwise by choosing a positive value for $R_{d}$, the poles can not be separated since $\mathbf{A} \neq \hat{\mathbf{A}}$. The position of the poles now depends on the system and also on the observer at the same time. Fig. 3 shows the poles position of the close-loop system dynamics for values of $R_{d}$ from $0 \Omega$ up to $20 \Omega$. For any value of the parameter $R_{d}$, the poles are always inside the unity circle, except for $R_{d}=0 \Omega$. This figure show the validity of our control algorithm and proves that with this proposal the system can be stabilized by using the SMC expressed in (43).
It is worth to mention that in filter-based active damping methods the stability analysis depends on the resonant frequency which largely depends on the grid impedance variations [14], [25]. As it can be seen in the previous analysis67, the roots placement does not rely on the grid impedance. This fact provides a high robustness against variations of this parameter, as will be shown in the experimental results section.

Although the system stability is independent of the grid impedance, the deviations of the LCL filter parameters should be studied. The most significative parameters to analyze the stability are the filter output inductor and the filter capacitor. Figs.4(a)-4(b) depict the root locus when the filter output inductor and the filter capacitor vary $\pm 30 \%$ with respect its nominal value. In this figure it can be seen that the stability analysis is assured for a large variations of these parameters.

\section{LCL FILTER DESIGN}

In order to design the LCL filter of the power converter, the procedure described in [26] has been adopted. This procedure has been applied to a power converter with a rated voltage of $E_{n}=190 V_{r m s}$ (line to line) and a rated power of $P_{n}=1.5$ $\mathrm{kW}$. For the switching frequency a typical value of $f_{s w}=10$ $\mathrm{kHz}$ has been selected, with a sampling frequency of $40 \mathrm{kHz}$. A switching algorithm is performed in order to achieve the desired switching frequency. The step-by-step procedure is as follows:

1) The first step is to calculate the base impedance and the base capacitor. The expressions for this purpose are expressed as follows:

$$
\begin{aligned}
Z_{b} & =\frac{E_{n}^{2}}{P_{n}}=12.1 \Omega \\
C_{b} & =\frac{1}{\omega_{g} Z_{b}}=219.22 \mu F
\end{aligned}
$$

2) The filter capacitor is designed according to the maximum power factor variation seen by the grid. Then, the selected reactive power absorbed at the rated conditions will determine the capacitor value. Considering a 5\% of the reactive power absorbed at rated conditions the maximum capacitor value is given by:

$$
C=0.05 C_{b}=8.7 \mu F
$$

which allows to use a nominal capacitor value of $C=6.8$ $\mu \mathrm{F}$

3) The maximum current ripple at the inverter-side inductor will determine the value of $L_{1}$. The current ripple is obtained as a percentage of the maximum current, in our case $10 \%$, (i.e $\Delta i_{1 i, \max }=0.1 i_{1 i, \max }$ ). Then, the value of $L_{1}$ is obtained as:

$$
L_{1}=\frac{V_{d c}}{6 f_{s w} \Delta i_{1 i, \max }}=1.6 m H
$$

4) Finally, the grid-side inductor, $L_{2}$, can be obtained as a ratio of the inductor $L_{1}$ as:

$$
L_{2}=r L_{1}
$$



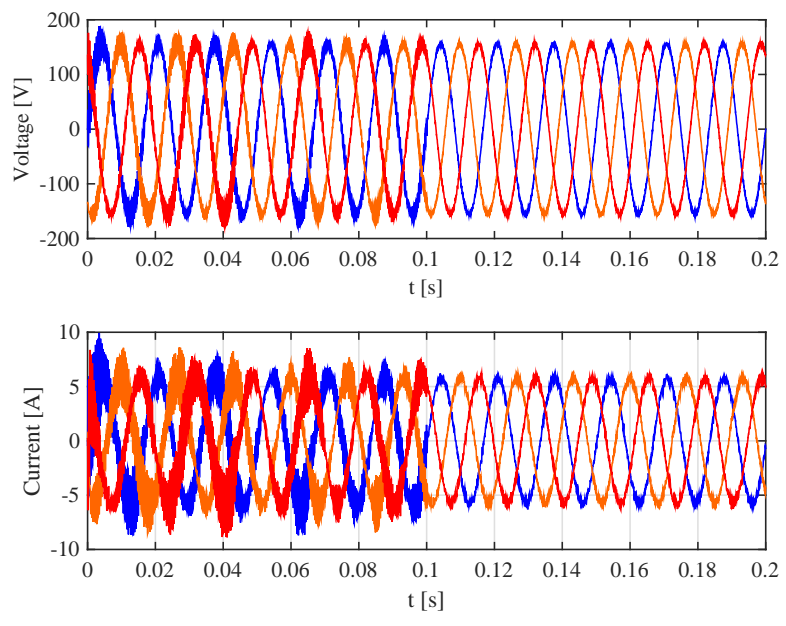

(a)
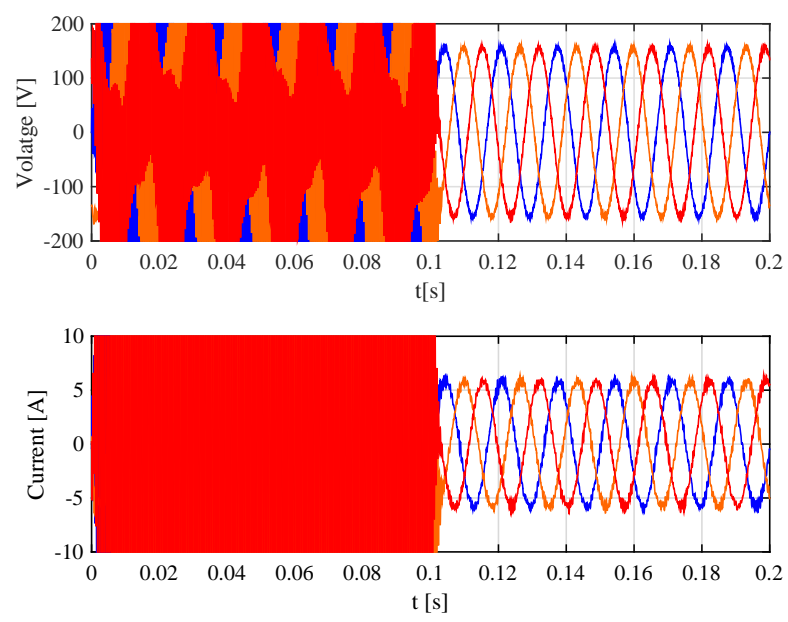

(b)
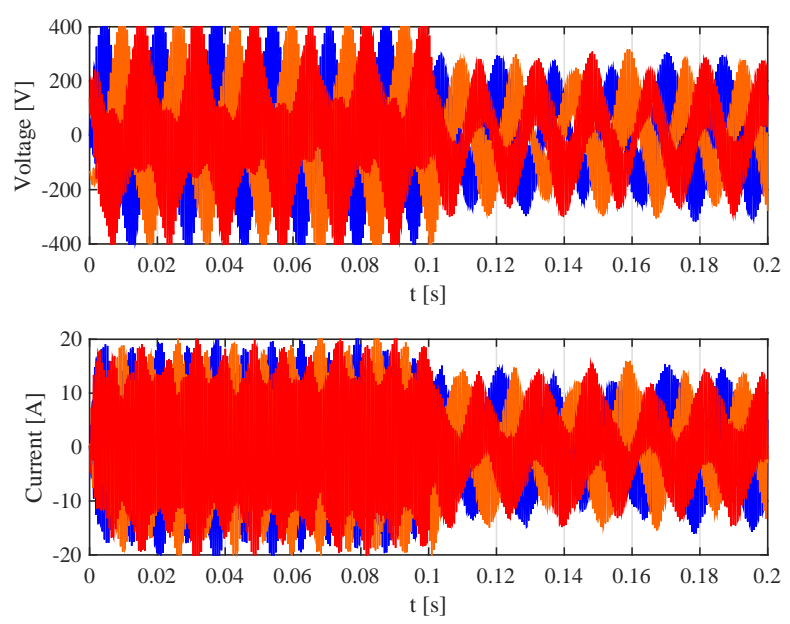

(c)

Fig. 5. Simulation results of PCC voltages and grid currents for three different values of $L_{g}$ : a) $L_{g}=0 \mathrm{mH}$, b) $L_{g}=0.5 \mathrm{mH}$, c) $L_{g}=1 \mathrm{mH}$.

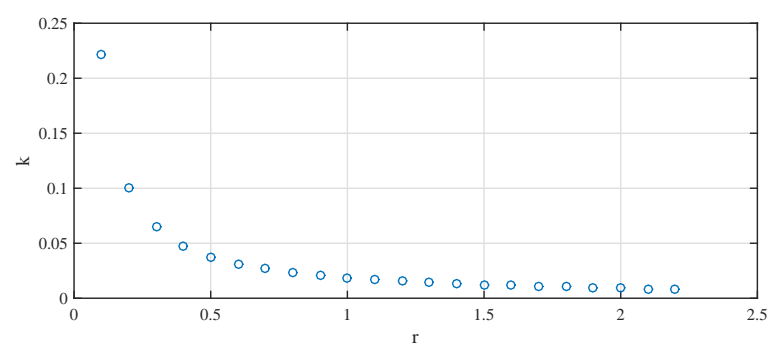

Fig. 6. Relation between ripple attenuation and the inductors ratio, r, at the switching frequency.

where the ratio $r$ depends of the desired ripple attenuation $k$. In our case a $20 \%$ of ripple attenuation is desired so that:

$$
k=\frac{1}{\left|1+r\left(1-L_{1} C_{b}\left(2 \pi f_{s w}\right)^{2}\right)\right|}=0.2
$$

Fig.6 represents the relation between the ripple attenuation and the ratio $r$ between both inductors. The figure shows that if a value of $r=0.1$ is taken, a ripple attenuation around $20 \%$ is obtained, as desired. So, the value of the inductor $L_{2}$ is $L_{2}=0.16 \mathrm{mH}$. According to 25 , this filter design results in a resonance frequency, $f_{r} e s$, around $1.8 \mathrm{kHz}$ when a grid inductance $L_{g}=0.5 \mathrm{mH}$ is considered. However, variations of the grid inductance will imply in variations of the resonance frequency.

\section{Simulation RESUlts USING THE VIRTUAL RESISTOR METHOD}

This section presents some simulation results of active damping based on a virtual resistor method. In addition, the controller bandwidth of the virtual resistor method will be compared with the control bandwidth of our proposal.

For this test, the system parameters are listed in in Table I. These results will be compared with the results obtained with the proposed controller. Three different values of the grid inductance are considered, $0 \mathrm{mH}, 0.5 \mathrm{mH}$ and $1 \mathrm{mH}$. Fig.5 shows the PCC voltages and the grid-side currents in three different scenarios. Fig.5(a) shows the case without grid inductance, $L_{g}=0 \mathrm{mH}$. At $\mathrm{t}=0.1 \mathrm{~s}$ the active damping is enabled, and the virtual resistor method works properly. In Fig.5(b) the grid inductance is $0.5 \mathrm{mH}$. As it can be seen, the active damping reduces the resonance but is not completely removed. Finally, in Fig.5(c), the grid inductance is incremented to $1 \mathrm{mH}$. It can be observed that in this case the method is not effective and the resonance can not be eliminated and the grid-side current is oscillating.

We can conclude that the virtual resistor method is not so effective compared with the proposed method, as it will be seen in the experimental results section.

\section{A. Controllers bandwidth comparison}

The controller bandwidth has been tested not only for the virtual resistor method, but also for the proposed control system. In order to find the controllers bandwidth, the frequency of the reference signal has been increased till a phase-lag of 30 

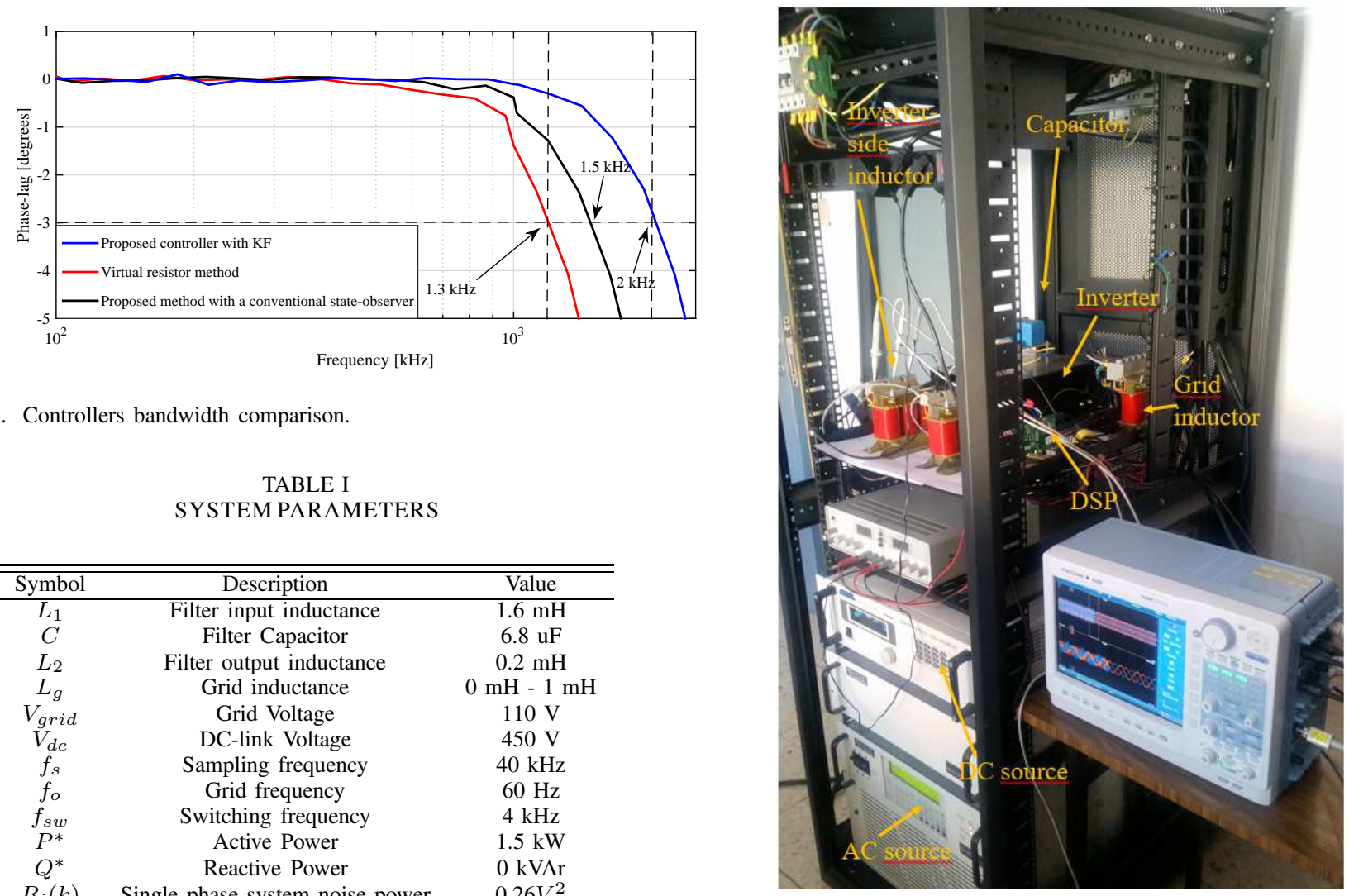

Fig. 7. Controllers bandwidth comparison.

TABLE I

SYSTEM PARAMETERS

\begin{tabular}{ccc}
\hline \hline Symbol & Description & Value \\
\hline$L_{1}$ & Filter input inductance & $1.6 \mathrm{mH}$ \\
$C$ & Filter Capacitor & $6.8 \mathrm{uF}$ \\
$L_{2}$ & Filter output inductance & $0.2 \mathrm{mH}$ \\
$L_{g}$ & Grid inductance & $0 \mathrm{mH}-1 \mathrm{mH}$ \\
$V_{g r i d}$ & Grid Voltage & $110 \mathrm{~V}$ \\
$V_{d c}$ & DC-link Voltage & $450 \mathrm{~V}$ \\
$f_{s}$ & Sampling frequency & $40 \mathrm{kHz}$ \\
$f_{o}$ & Grid frequency & $60 \mathrm{~Hz}$ \\
$f_{s w}$ & Switching frequency & $4 \mathrm{kHz}$ \\
$P^{*}$ & Active Power & $1.5 \mathrm{~kW}$ \\
$Q^{*}$ & Reactive Power & $0 \mathrm{kVAr}$ \\
$R_{i}(k)$ & Single phase system noise power & $0.26 V^{2}$ \\
$Q_{i}(k)$ & Process covariance matrix & $0.005 \mathbf{I}_{5}$ \\
\hline \multicolumn{3}{c}{} \\
\hline
\end{tabular}

between the generated current and its reference is measured. This method is widely explained in [17].

It has been found that, for a switching frequency of 10 $\mathrm{kHz}$, the bandwidth of the virtual method is around $1.3 \mathrm{kHz}$ while with the proposed control algorithm with a switching frequency of $4 \mathrm{kHz}$ is around $2 \mathrm{kHz}$, as shown Fig.7. The reason is that the oversampling, and the use of the KF which increases significantly the SNR of the system, provides an important reduction of switching losses. This is a superior feature of this control proposal.

\section{EXPERIMENTAL VALIDATION}

Fig. 8 shows the experimental three-phase three-wire inverter prototype using a $4.5-\mathrm{kVA}$ SEMIKRON full-bridge as the power converter, and a TMS320F28M36 floating-point digital signal processor (DSP) as the control platform with a sampling frequency of $40 \mathrm{kHz}$. The grid and the DC-link voltages have been generated using a PACIFIC 360-AMX and an AMREL SPS1000-10-K0E3 sources respectively. The system parameters are listed in Table I. Some experimental results has been exported to MATLAB $\subset$ for the final representation.

Using the approximations reported in section III-B, the computational load of the KF algorithm has been reduced from $14 \mu \mathrm{s}$ to $5 \mu \mathrm{s}$. Note that in each phase-leg a different $\mathrm{KF}$ is used but the Kalman gain is the same for each phaseleg. This is an important reduction in the total algorithm time which now is around of $24 \mu \mathrm{s}$. This fact allows a sampling frequency $f_{s}=40 \mathrm{kHz}$ and makes possible the implementation of this proposal in the DSP. The switching frequency, $f_{s w}$ when sliding mode control is used can be chosen as $f_{s w}=f_{s} / 10$ to ensure enough samples for a proper performance of the variable hysteresis comparator. This ratio provides a good trade-off between the use of the computational load in a switching period and the switching frequency accuracy. The following figures show the good performance of the proposed control system.

\section{A. Model parameters mismatch}

Fig.9 shows the grid-side currents in the case of parameters mismatch in the model. In 9(a) the model parameters have been reduced to $100 \%$ of its nominal values, while in Fig.9(b) the values have been reduced to $100 \%$ of the nominal values. If both signals are compared, a reduction in the current ripple can be observed without affecting to the correct controller operation. Thanks to this property, the value of the inductor $L_{1}$ can be incremented in the model in order to reduce the current ripple without changing the LCL system parameters. This fact allows to select lower switching frequencies using smaller LCL filters.

\section{B. Test of robustness against grid inductance variations}

In order to compare the results of the proposed control method with the results obtained in the previous section, the following figures are shown. Fig.10 shows a test of robustness against grid inductance variations. In order to reduce the 


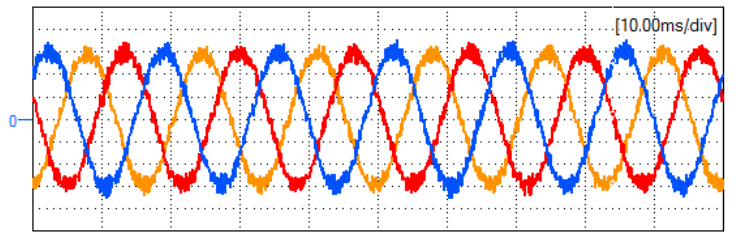

(a)

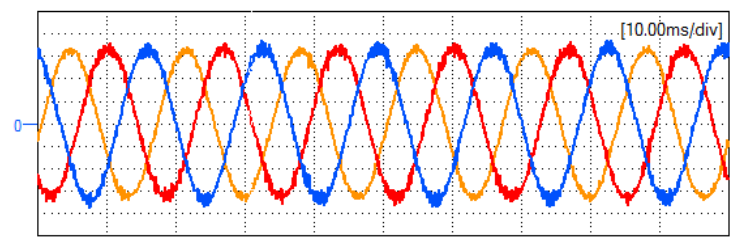

(b)

Fig. 9. Grid-side currents ( $2 \mathrm{~A} / \mathrm{div}, 10 \mathrm{~ms} / \mathrm{div}$ ) for two different values of $L_{1}$ in the converter model :a) $L_{1}=1.6 \mathrm{mH}$ and b) $L_{1}=7 \mathrm{mH}$.

current ripple without the necessity of increasing the switching frequency, the inductor value $L_{1 o}$ of the proposed converter model has been changed to $7 \mathrm{mH}$ while the converter physical inductor is maintained to $1.6 \mathrm{mH}$. Note that, the LCL filter parameters have been designed for a switching frequency of $10 \mathrm{kHz}$ but the SMC operates at a switching frequency of 4 $\mathrm{kHz}$.

The active damping has been tested for three different values of $L_{g}: 0 \mathrm{mH}, 0.5 \mathrm{mH}$ and $1 \mathrm{mH}$, respectively. The PCC voltages and the grid-side currents are shown for these three different scenarios. In these tests, two different values of $R_{d}$ are used, being $R_{d}=0 \Omega$ when active damping is disabled, and $R_{d}=10 \Omega$ when active damping is enabled. The results of these tests are discussed as follows: 1) Fig.10(a) shows the PCC voltages and the grid-side currents when the nominal grid inductance is $L_{g}=0 \mathrm{mH}$. As it can be seen, when the active damping is disabled the system is oscillating since the controller (43) is working with a virtual damping resistor $R_{d}=0 \Omega$. When the active damping is enabled the system is stabilized and all the roots are attracted inside the unity circle in the z-plane, as shown in Fig.3.

2) The same test is shown in Fig.10(b) but in this case the grid inductance is incremented to a value of $L_{g}=0.5 \mathrm{mH}$. As it can be seen the control algorithm works as expected, and the oscillation disappears when the active damping is enabled.

3) Finally, in Fig.10(c) the grid inductance is $L_{g}=1 \mathrm{mH}$. Once again, the same satisfactory results are obtained. We can conclude that the effectiveness of the control algorithm is practically independent of the grid inductance variations. This is a superior feature of the proposed control in comparison with filter-based active damping.

Compared with the case of the virtual resistor method, shown in section VII, similar results are obtained using a reduced switching frequency. It can be achieved without increasing the size of the LCL filter parameters.

\section{Controller dynamic response under a sudden current step change}

Fig.11 shows the transient behaviour against changes in the reference current. In order to reduce the current ripple, the

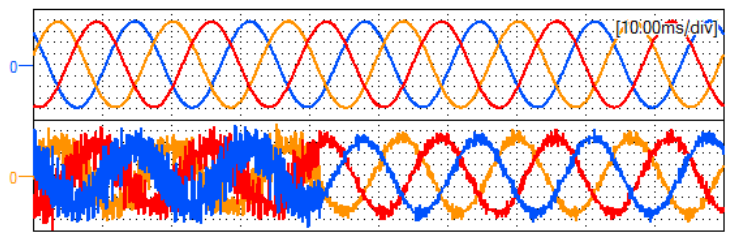

(a)

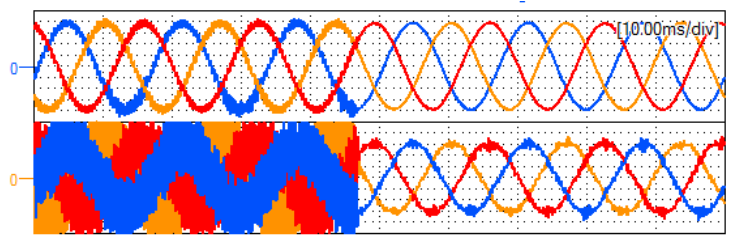

(b)

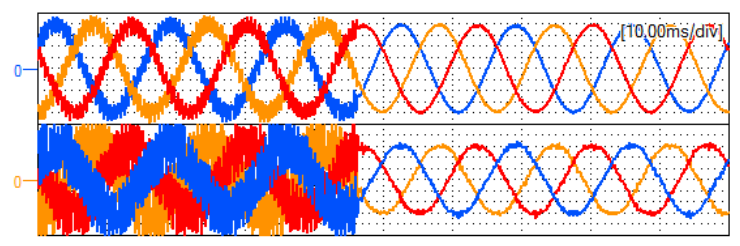

(c)

Fig. 10. PCC voltages $(50 \mathrm{~V} / \mathrm{div})$ and grid currents $(2 \mathrm{~A} / \mathrm{div}, 20 \mathrm{~ms} / \mathrm{div})$ for three different values of $L_{g}$ : a) $L_{g}=0 \mathrm{mH}$, b) $L_{g}=0.5 \mathrm{mH}$, c) $L_{g}=1 \mathrm{mH}$.

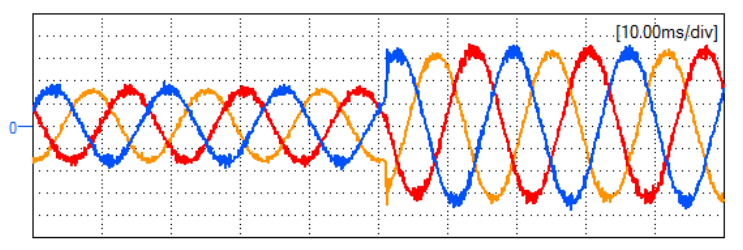

Fig. 11. Grid-side currents (2 A/div, $10 \mathrm{~ms} / \mathrm{div})$.

value of the input inductor $L_{1 o}$ in the proposed model is changed to $7 \mathrm{mH}$, while the value of $L_{1}$ in the system is maintained to $1.6 \mathrm{mH}$. A reference current step change from $3.1 \mathrm{~A}$ to $6.2 \mathrm{~A}$ is applied. As it can be seen a fast transient response is achieved.

\section{Test of the VSI under distorted grid}

Fig.12 compares the three-phase grid currents using the conventional SMC (17) and using the proposed control method in the case of a distorted grid. This figure shows the distorted PCC voltages, which Total Harmonic Distortion (THD) is around $14 \%$, and the grid-side currents. As shown in the figure, when the conventional SMC is used, the grid currents are distorted since the reference currents generation method, (18)-(20), uses distorted PCC voltages. In contrast, when the proposed control method is used, the grid currents are practically sinusoidal since the reference current is generated by using only the fundamental component of the PCC voltage, (44)-(46), obtained from the KF. Note that the method uses the estimated PCC voltage obtained from the KF which are computed using the measured inverter-side current. For this reason these voltages are in phase with the real ones but without harmonics and noise. Thanks to this approach it is 


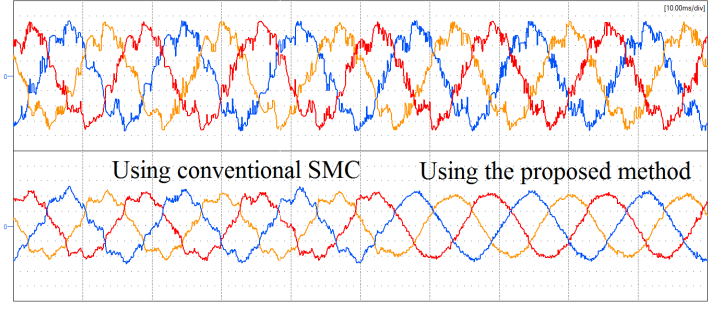

Fig. 12. Distorted PCC voltages (25V/div) with $\mathrm{THD}=14 \%$, and grid-side currents $(5 \mathrm{~A} / \mathrm{div})$.

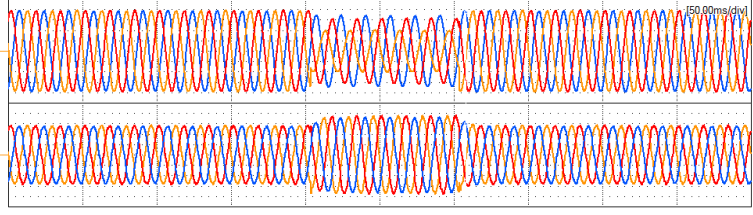

Fig. 13. PCC voltages $(50 \mathrm{~V} /$ div $)$ and grid-side currents $(2 \mathrm{~A} / \mathrm{div})$ under voltage sag.

not necessary to use any mechanism of synchronization as for example a Phase Locked Loop (PLL), obtaining the same satisfactory results (please see [20], [21]. This is one of the advantages of this control method.

Note that with this proposal, the use of extra filters for the grid voltages are not necessary.

\section{E. Test of VSI under voltage sags}

Fig.13 shows the VSI performance under grid voltage sags. A two-phase fault is analyzed in this test. The reference currents can be obtained in this case using the positive sequence of the PCC voltage, using $\hat{i}_{a, b, c}^{*}=\frac{P^{*}}{|\hat{\mathbf{v}}|^{2}} \hat{v}_{a, b, c}^{+}$. The positive sequence can be computed using the equations presented in the Appendix. In these equations the estimated PCC voltages are used. These voltages are estimated using the Kalman estimator (39) which uses the measured inverter side current in its computation. Note that the frequency and phase information is contained in this variable. For this reason, the use of a specific PLL algorithm for extracting the positive and negative sequence grid voltage components is not necessary. Due to the fact that the grid current tracks only the positive sequence of the PCC voltage, the current amplitude is the same in the three phases during the voltage sag.

\section{F. Control signal frequency spectrum}

In Fig.14(a) the sliding surfaces with its corresponding control signals in the case of coupled controllers are shown. In this case the three controllers are implemented with measured variables instead of estimated ones. The figure shows the effect of the neutral point voltage (6) in the controllers dynamics. As it can be seen the slope of each sliding surface change with any of the control signals losing the synchronization and making impossible to design a current control with variable hysteresis band operating at fixed switching frequency.

In Fig.14(b) the sliding surfaces with its corresponding control signals in the case of decoupled controllers are shown. In this case the sliding mode controllers uses the estimated

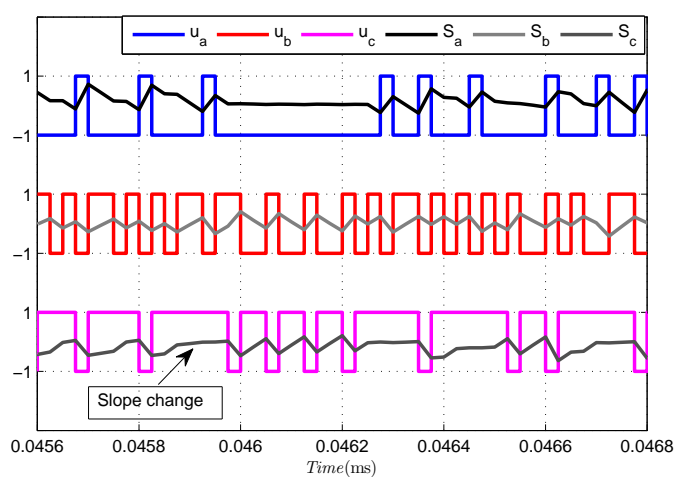

(a)

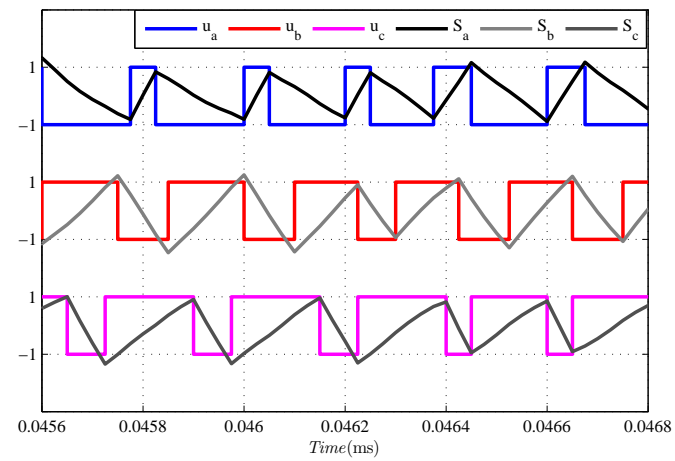

(b)

Fig. 14. Experimental control signals $u_{a}, u_{b}$ and $u_{c}$ with its corresponding sliding surfaces $S_{a}, S_{b}$ and $S_{c}$ (a) with coupled controllers, (b) with decoupled controllers operating at fixed switching frequency.

inverter-side currents provided by (39). As it can be seen the axis-decoupling is effective, and the slope of each sliding surface only changes with its control variable. Using the proposed model the three sliding surfaces are independent of each others and the variable hysteresis control can be performed ensuring a fixed switching frequency.

Fig.15(a) shows the transient performance of the variable hysteresis band regulator for phase-leg $a$ without using the switching decision algorithm defined in section IV-D. From this figure, it can be seen that some samples of the sliding surface are out of the hysteresis band limits. This fact provokes an error in the desired switching frequency. In order to improve the variable hysteresis band controllers and also eliminate this error, the switching decision algorithm is introduced. The effect of this algorithm is shown in Fig.15(b). As it can be seen the number of samples that appears out of the hysteresis band limits is reduced and the effective switching frequency is increased eliminating the aforementioned error.

Fig.16(a) shows the control signals spectrum when the variable hysteresis band is used. The spectrum is concentrated around a frequency lower than the desired switching frequency of $4 \mathrm{kHz}$. To avoid this problem the switching decision algorithm is used and the switching frequency is now concentrated at $4 \mathrm{kHz}$ as shown Fig.16(b). 


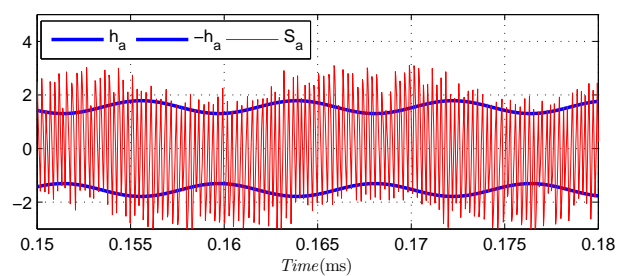

(a)

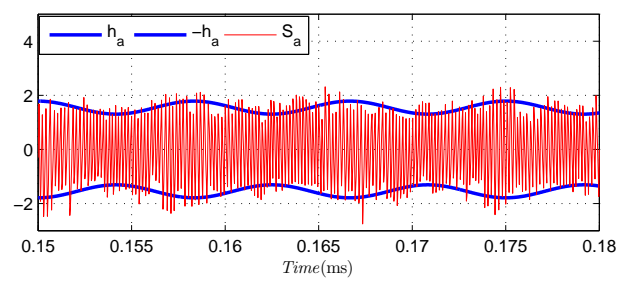

(b)

Fig. 15. Experimental hysteresis band and its switching surface for phase-leg $a$ : (a) without switching algorithm [21], (b) with switching algorithm [21].

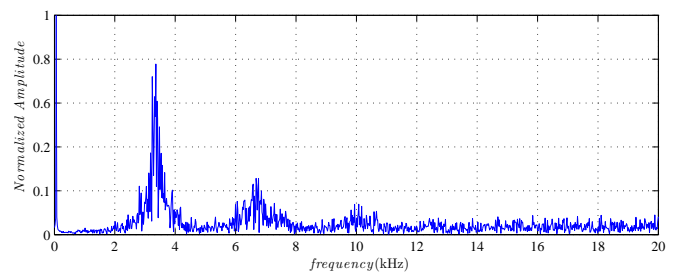

(a)

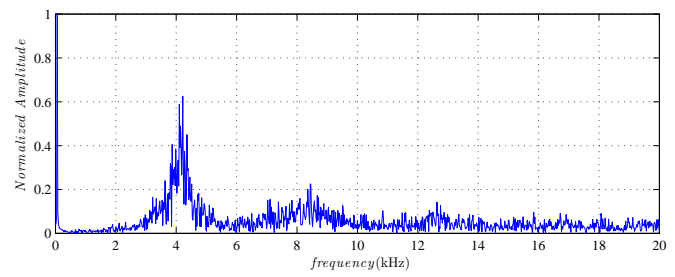

(b)

Fig. 16. Switching signal spectrum: (a) using variable hysteresis band control but without switching algorithm [21], (b) using variable hysteresis band control and switching algorithm [21].

\section{CONClusions}

In this paper a new concept in active damping methods has been presented. The proposed method uses a model-based solution in natural frame for a three-phase voltage source inverter with LCL filter. The proposed method is based on introducing a virtual damping resistor in series with the filter capacitor in the converter model. This model is the basis of a Kalman filter used to obtain the estimated currents which allow us to derive three current sliding-mode controllers in natural frame. The use of estimated variables instead of the measured ones provide the controllers with active damping capability, independent of the system parameters. This solution leads to a system behaviour identical as when a physical damping resistor is used but with the difference that system losses are avoided. Besides, the use of a Kalman filter instead of a non-adaptive state observer improves the quality of the estimated signals in presence of noise increasing the noise immunity of the control loop. The control algorithm has been implemented in a DSP working at a sampling frequency of $40 \mathrm{kHz}$, which limits the fixed switching frequency to $4 \mathrm{kHz}$. For this reason, a useful approximation has been used to reduce the computational time needed for the $\mathrm{KF}$ implementation reducing the total time employed for the control algorithm. Note that, the switching frequency could be increased if a faster digital signal processor or a FPGA is used. The stability analysis and the corresponding experimental results shows the validity of this proposal. In future work, this technique can be applied to other converters using the appropriate converter model.

\section{APPENDIX}

Defining the Clarke transformation as:

$$
\left[T_{\alpha \beta}\right]=\sqrt{\frac{2}{3}}\left[\begin{array}{ccc}
1 & -\frac{1}{2} & -\frac{1}{2} \\
0 & \frac{\sqrt{3}}{2} & -\frac{\sqrt{3}}{2}
\end{array}\right]
$$

and applying the transformation to the estimated PCC voltages and their quadratures, yields:

$$
\begin{gathered}
{\left[\begin{array}{c}
\hat{v}_{\alpha} \\
\hat{v}_{\beta}
\end{array}\right]=\left[T_{\alpha \beta}\right]\left[\begin{array}{l}
\hat{v}_{a} \\
\hat{v}_{b} \\
\hat{v}_{c}
\end{array}\right]} \\
{\left[\begin{array}{c}
\hat{v}_{\alpha q} \\
\hat{v}_{\beta q}
\end{array}\right]=\left[T_{\alpha \beta}\right]\left[\begin{array}{l}
\hat{v}_{a q} \\
\hat{v}_{b q} \\
\hat{v}_{c q}
\end{array}\right] .}
\end{gathered}
$$

Computation of the estimated positive sequence, $\hat{v}_{\alpha}^{+}$and $\hat{v}_{\beta}^{+}$:

$$
\begin{aligned}
& \hat{v}_{\alpha}^{+}=\frac{1}{2} \hat{v}_{\alpha}-\frac{1}{2} \hat{v}_{\beta q} \\
& \hat{v}_{\beta}^{+}=\frac{1}{2} \hat{v}_{\alpha q}+\frac{1}{2} \hat{v}_{\beta} .
\end{aligned}
$$

Anti Clarke transformation to obtain the positive sequence of the estimated voltage at the PCC in $a b c$ frame:

$$
\left[\begin{array}{c}
\hat{v}_{a}^{+} \\
\hat{v}_{b}^{+} \\
\hat{v}_{c}^{+}
\end{array}\right]=\left[T_{\alpha \beta}\right]^{T}\left[\begin{array}{l}
\hat{v}_{\alpha}^{+} \\
\hat{v}_{\beta}^{+}
\end{array}\right] .
$$

\section{REFERENCES}

[1] M. Cespedes, L. Xing, and J. Sun, "Constant-power load system stabilization by passive damping," IEEE Trans. Power Electron., vol. 26, no. 7, pp. 1832-1836, 2011.

[2] R. Peña Alzola, M. Liserre, F. Blaabjerg, R. Sebastian, J. Dannehl, and F. Fuchs, "Analysis of the passive damping losses in LCL-filter-based grid converters," IEEE Trans. Power Electron., vol. 28, no. 6, pp. 26422646, 2013.

[3] K. Hatua, A. Jain, D. Banerjee, and V. Ranganathan, "Active damping of output lc filter resonance for vector-controlled VSI-fed ac motor drives," IEEE Trans. Ind. Electron., vol. 59, no. 1, pp. 334-342, Jan 2012.

[4] M. Hanif, V. Khadkikar, W. Xiao, and J. Kirtley, "Two degrees of freedom active damping technique for LCL filter-based grid connected pv systems," IEEE Trans. Ind. Electron., vol. 61, no. 6, pp. 2795-2803, June 2014.

[5] E. Figueres, G. Garcera, J. Sandia, F. Gonzalez-Espin, and J. Rubio, "Sensitivity study of the dynamics of three-phase photovoltaic inverters with an LCL grid filter,' IEEE Trans. Ind. Electron., vol. 56, no. 3, pp. 706-717, 2009. 
[6] M. Liserre, F. Blaabjerg, and S. Hansen, "Design and control of an 1clfilter-based three-phase active rectifier," IEEE Transactions on Industry Applications, vol. 41, no. 5, pp. 1281-1291, Sept 2005.

[7] R. Guzman, L. G. de Vicua, J. Morales, A. Momeneh, J. Miret, and J. Torres-Martnez, "Active damping based on ackermann's formula for a three-phase voltage source inverter with lcl filter," in Industrial Electronics Society, IECON 2015 - 41st Annual Conference of the IEEE, Nov 2015, pp. $001217-001222$.

[8] X. Wang, F. Blaabjerg, and P. C. Loh, "Grid-current-feedback active damping for LCL resonance in grid-connected voltage-source converters," IEEE Trans. Power Electron., vol. 31, no. 1, pp. 213-223, Jan 2016.

[9] D. Pan, X. Ruan, C. Bao, W. Li, and X. Wang, "Capacitor-currentfeedback active damping with reduced computation delay for improving robustness of LCL-type grid-connected inverter," IEEE Trans. Power Electron., vol. 29, no. 7, pp. 3414-3427, July 2014

[10] F. Liu, B. Wu, N. Zargari, and M. Pande, "An active damping method using inductor-current feedback control for high-power PWM currentsource rectifier," IEEE Trans. Power Electron., vol. 26, no. 9, pp. 25802587, 2011.

[11] C. Wessels, J. Dannehl, and F. Fuchs, "Active damping of LCLfilter resonance based on virtual resistor for pwm rectifiers - stability analysis with different filter parameters," in Power Electron. Specialists Conference, 2008. PESC 2008. IEEE, June 2008, pp. 3532-3538.

[12] J. Dannehl, M. Liserre, and F. Fuchs, "Filter-based active damping of voltage source converters with LCL filter," IEEE Trans. Ind. Electron., vol. 58, no. 8, pp. 3623-3633, 2011.

[13] M. Liserre, A. Aquila, and F. Blaabjerg, "Genetic algorithm-based design of the active damping for an LCL-filter three-phase active rectifier," IEEE Trans. Power Electron., vol. 19, no. 1, pp. 76-86, 2004.

[14] R. Peña Alzola, M. Liserre, F. Blaabjerg, M. Ordonez, and Y. Yang, "LCL-filter design for robust active damping in grid-connected converters," IEEE Trans. Ind. Inf., vol. 10, no. 4, pp. 2192-2203, Nov 2014.

[15] R. Peña Alzola, M. Liserre, F. Blaabjerg, M. Ordonez, and T. Kerekes, "A self-commissioning notch filter for active damping in a three-phase LCL -filter-based grid-tie converter," IEEE Trans. Power Electron., vol. 29, no. 12, pp. 6754-6761, Dec 2014.

[16] R. Peña Alzola, M. Liserre, F. Blaabjerg, R. Sebastian, J. Dannehl, and F. Fuchs, "Systematic design of the lead-lag network method for active damping in LCL-filter based three phase converters," IEEE Trans. Ind. Inf., vol. 10, no. 1, pp. 43-52, Feb 2014.

[17] S. Buso and T. Caldognetto, "A nonlinear wide-bandwidth digital current controller for DC-DC and DC-AC converters," IEEE Trans. on Ind. Electron., vol. 62, no. 12, pp. 7687-7695, Dec 2015.

[18] X. Zhang and Z. Li, "Sliding mode observer-based mechanical parameter estimation for permanent-magnet synchronous motor," IEEE Trans. Power Electron., vol. PP, no. 99, pp. 1-1, 2015.

[19] R. Guzman, L. Garcia de Vicuna, A. Camacho, J. Matas, M. Castilla, and J. Miret, "Active damping control for a three phase grid-connected inverter using sliding mode control," in Ind. Electron. Society, IECON 2013 - 39th Annual Conference of the IEEE, Nov 2013, pp. 382-387.

[20] J. Kanieski, R. Cardoso, H. Pinheiro, and H. Grundling, "Kalman filterbased control system for power quality conditioning devices," IEEE Trans. Ind. Electron., vol. 60, no. 11, pp. 5214-5227, Nov 2013.

[21] R. Guzman, L. G. de Vicuna, J. Morales, M. Castilla, and J. Matas, "Sliding-mode control for a three-phase unity power factor rectifier operating at fixed switching frequency," IEEE Trans. Power Electron., vol. 31, no. 1, pp. 758-769, Jan 2016.

[22] M. L. W. Gene F. Franklin, J. David Powell, Digital Control of Dynamic Systems. Addison-Wesley, 1997.

[23] X. Quingsong and T. Kiong, Advanced Control of Piezoelectric Micro/Nano-Positioning Systems. Springer, 2015.

[24] Q. Xu and Y. Li, "Model predictive discrete-time sliding mode control of a nanopositioning piezostage without modeling hysteresis," IEEE Trans. Control Systems Technology, vol. 20, no. 4, pp. 983-994, July 2012.

[25] S. Parker, B. McGrath, and D. Holmes, "Regions of active damping control for LCL filters," IEEE Trans. Ind. Applications., vol. 50, no. 1, pp. 424-432, Jan 2014.

[26] M. Liserre, F. Blaabjerg, and S. Hansen, "Design and control of an lcl-filter-based three-phase active rectifier," IEEE Trans. on Ind. Appl., vol. 41, no. 5, pp. 1281-1291, Sept 2005.

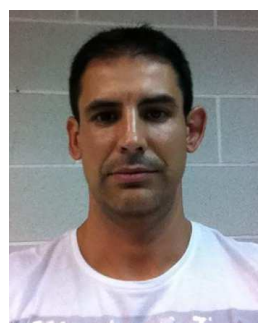

Ramon Guzman received the B.S. and M.S. degrees in telecommunications engineering from the Technical University of Catalonia, Barcelona, Spain, in 1999 and 2004, respectively. He is currently working toward the Ph.D. degree in model-based control of power converters, in the Power and Control Electronics Systems Group. Since 2001, he has been an Associate Professor with the Department of Communications and Signal Theory, Technical University of Catalonia, Barcelona, Spain. His current research interests are in the area of nonlinear and adaptive control for three-phase power converters.

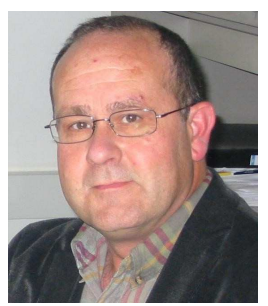

Luis Garcia de Vicuña received the M.S. and Ph.D. degrees in telecommunication engineering from the Technical University of Catalonia, Barcelona, Spain, in 1980 and 1990, respectively, and the Ph.D. degree in electrical engineering from the Paul Sabatier University, Toulouse, France, in 1992. From 1980 to 1982, he was an Engineer with a control applications company in Spain. He is currently a Full Professor with the Department of Electronic Engineering, Technical University of Catalonia, where he teaches courses on power electronics. His research interests include power electronics modeling, simulation and control, active power filtering, and high-power-factor ac/dc conversion.

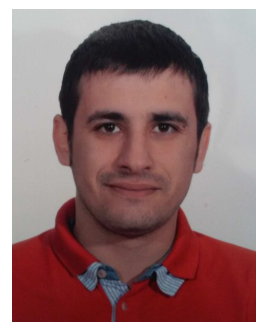

Javier Morales received the B.S. degree in electronics engineering and the M.S. degree in automation and industrial electronics from the Technical University of Catalonia, Barcelona, Spain, in 2012 and 2014, respectively. Currently, he is working towards the Ph.D. degree in electronic engineering at the Technical University of Catalonia. His research interests include automatic control and power electronics.

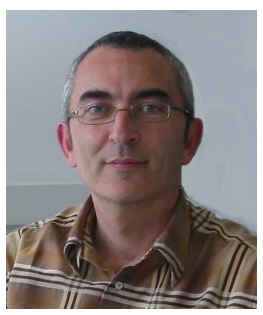

Miguel Castilla received the B.S., M.S. and Ph.D. degrees in telecommunication engineering from the Technical University of Catalonia, Barcelona, Spain, in 1988,1995 , and 1998, respectively. Since 2002, he has been an Associate Professor in the Department of Electronic Engineering, Technical University of Catalonia, where he teaches courses on analog circuits and power electronics. His research interests are in the areas of power electronics, nonlinear control, and renewable energy systems.

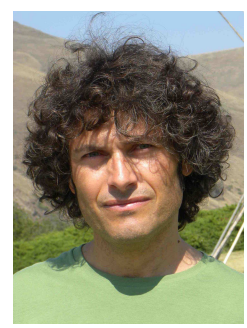

Jaume Miret (M98) received the B.S. degree in telecommunications, M.S. degree in electronics, and Ph.D. degree in electronics from the Universitat Politecnica de Catalunya, Barcelona, Spain, in 1992, 1999, and 2005, respectively. From 1993 to 2011, he was an Assistant Professor in the Department of Electronic Engineering, Universitat Politecnica de Catalunya, Spain. Since 2011 he has been an Associate Professor in the Universitat Politecnica de Catalunya, where he teaches courses on digital design and circuit theory. His research interests include dc-to-ac converters, active power filters, and digital control 